\title{
A Spectrum Handoff Scheme for Optimal Network Selection in NEMO Based Cognitive Radio Vehicular Networks
}

\author{
Krishan Kumar, ${ }^{1,2}$ Arun Prakash, ${ }^{1}$ and Rajeev Tripathi ${ }^{1}$ \\ ${ }^{1}$ Department of Electronics and Communication Engineering, Motilal Nehru National Institute of Technology, Allahabad, India \\ ${ }^{2}$ Electronics and Communication Engineering Department, National Institute of Technology, Hamirpur, India \\ Correspondence should be addressed to Krishan Kumar; krishan_rathod@nith.ac.in
}

Received 30 July 2016; Accepted 3 October 2016; Published 10 January 2017

Academic Editor: Antonio de la Oliva

Copyright (c) 2017 Krishan Kumar et al. This is an open access article distributed under the Creative Commons Attribution License, which permits unrestricted use, distribution, and reproduction in any medium, provided the original work is properly cited.

\begin{abstract}
When a mobile network changes its point of attachments in Cognitive Radio (CR) vehicular networks, the Mobile Router (MR) requires spectrum handoff. Network Mobility (NEMO) in CR vehicular networks is concerned with the management of this movement. In future NEMO based CR vehicular networks deployment, multiple radio access networks may coexist in the overlapping areas having different characteristics in terms of multiple attributes. The CR vehicular node may have the capability to make call for two or more types of nonsafety services such as voice, video, and best effort simultaneously. Hence, it becomes difficult for MR to select optimal network for the spectrum handoff. This can be done by performing spectrum handoff using Multiple Attributes Decision Making (MADM) methods which is the objective of the paper. The MADM methods such as grey relational analysis and cost based methods are used. The application of MADM methods provides wider and optimum choice among the available networks with quality of service. Numerical results reveal that the proposed scheme is effective for spectrum handoff decision for optimal network selection with reduced complexity in NEMO based CR vehicular networks.
\end{abstract}

\section{Introduction}

With the exponential growth in the number of vehicles on the road, there is need of extensive research to improve the spectrum efficiency for improving vehicular communications. The significant rise in the vehicular communication applications may lead to the overcrowding of the allocated spectrum bands, resulting in degraded vehicular communication efficiency. It has raised the demand of extra spectrum bands to solve the problems of spectrum scarcity. Due to static spectrum band allocation policy by the government agencies, some of the allocated spectrum bands are underutilized such as some portion of TV VHF/UHF bands, so-called TV white spaces [1-4]. To solve the problems of spectrum scarcity, US Federal Communication Commission (FCC) has suggested to use the underutilized spectrums and spectrum potions which are not in use, called vacant spectrum bands or white spaces [3]. This generates a new area of research and development of Cognitive Radio (CR) technology through which unlicensed devices, called CR, can use the licensed unutilized spectrum bands [4-9]. CR networks are receiving significant attention from the researchers to overcome the apparent spectrum scarcity problem, as well as to improve the communication efficiency $[4,6,10]$. Hence, it is envisioned that the future vehicular communications will be CR enabled which can use additional spectrum opportunities to improve vehicular communication efficiency [11]. In future CR vehicular networks deployment, there is high communication demand during mobility. Therefore, the node and network mobility are the important features in vehicular communications. The network mobility arises when "collective mobility of a group of nodes [Network Mobility (NEMO)]" comes into the picture $[11,12]$. For example, people inside trains, buses, planes, and ships accessing wireless networks nonsafety services such as voice, video, and best effort services. When a mobile network changes its point of attachment in CR vehicular networks, NEMO is concerned with the management of this movement which can be done by the spectrum handoff as shown in Figure 1. Figure 1 presents the system model showing different types of spectrum handoffs in NEMO based $\mathrm{CR}$ vehicular networks. It is shown that the intranetwork spectrum handoff takes place within the same radio access 


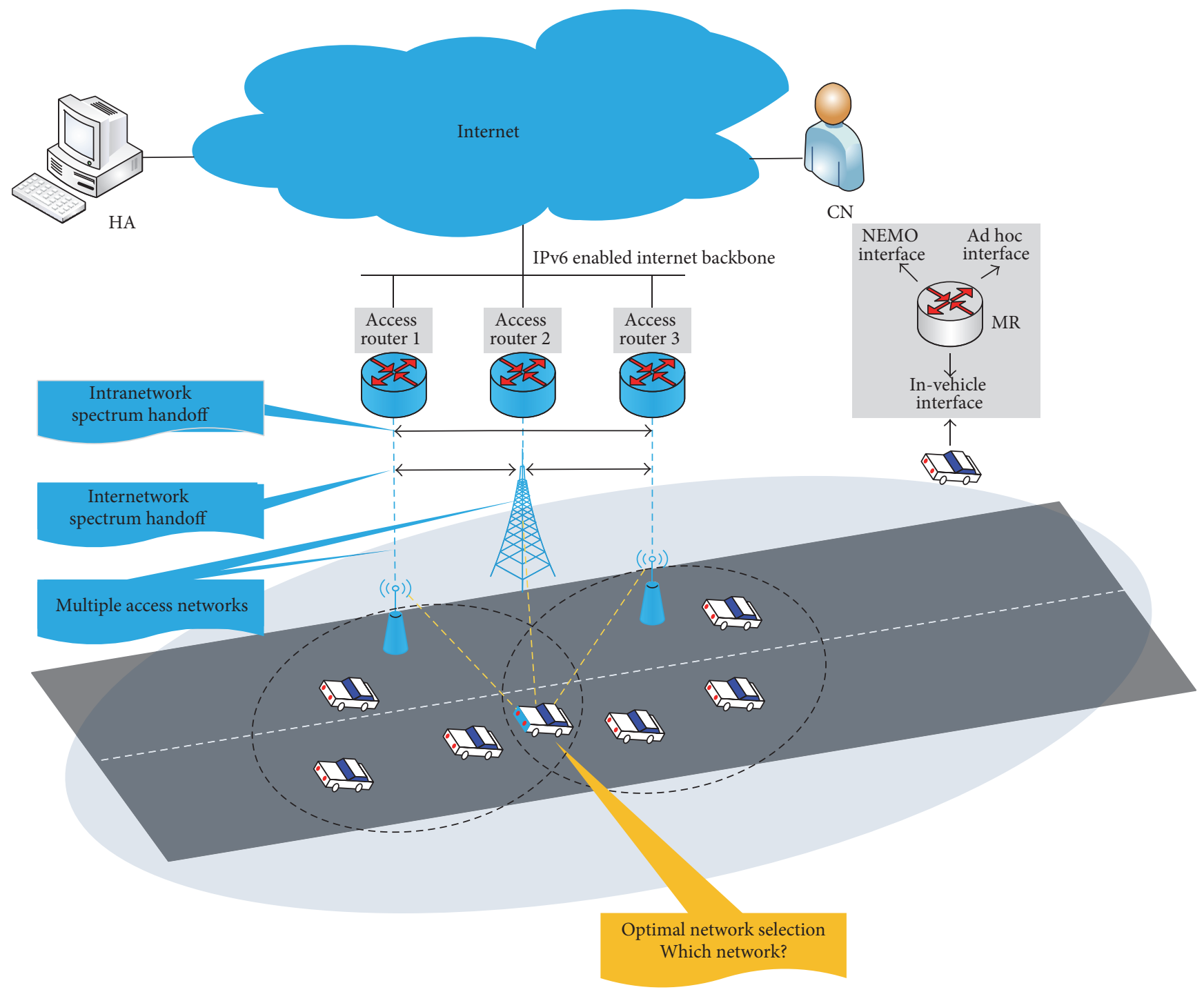

FIGURE 1: System model showing spectrum handoff in NEMO based CR vehicular networks.

technology whereas internetwork spectrum handoff takes place between the heterogeneous radio access technologies. Figure 1 also shows that some CR vehicular nodes called Mobile Routers (MRs) are connected with one radio access technology and some are connected with multiple radio access technologies. When MR is connected with multiple radio access technologies, then it is not easy for the MR to select the optimal network for the spectrum handoff decision. Figure 2 shows that in future CR vehicular networks deployment multiple radio access networks may coexist in the overlapping areas having different characteristics in terms of multiple attributes. The CR vehicular node, termed as multimode $\mathrm{CR}$ vehicular node, may have the capability to make call for two or more types of nonsafety services, such as voice, video, and best effort services simultaneously. Hence, it becomes difficult for MR to select optimal network for the spectrum handoff in such scenarios. This can be done by spectrum handoff scheme using multiple attributes decision making (MADM) for optimal network selection.
This motivates us to design spectrum handoff scheme using MADM methods for optimal network selection in NEMO based CR vehicular networks. The MADM methods used are grey relational analysis (GRA) [13-15] and cost based method [16]. The advantages of GRA method are that the results are based on the original data and numerical calculations are easy and simple [14]. The advantages of cost based method are that the multiple attributes are used directly for the estimation of cost. Therefore, it is very simple and one of the best method to make decision under heterogeneous network environment [16]. The MADM methods have the property to make decision for selecting an appropriate network from a predetermined number of available networks having white spaces. The MADM methods provide optimized results with reduced complexity problem in spectrum handoff. The safety and nonsafety services are two types of services in vehicular communication. This work does not considered safety services which are the essential service in vehicular communication. Whenever spectrum handoff is required for 


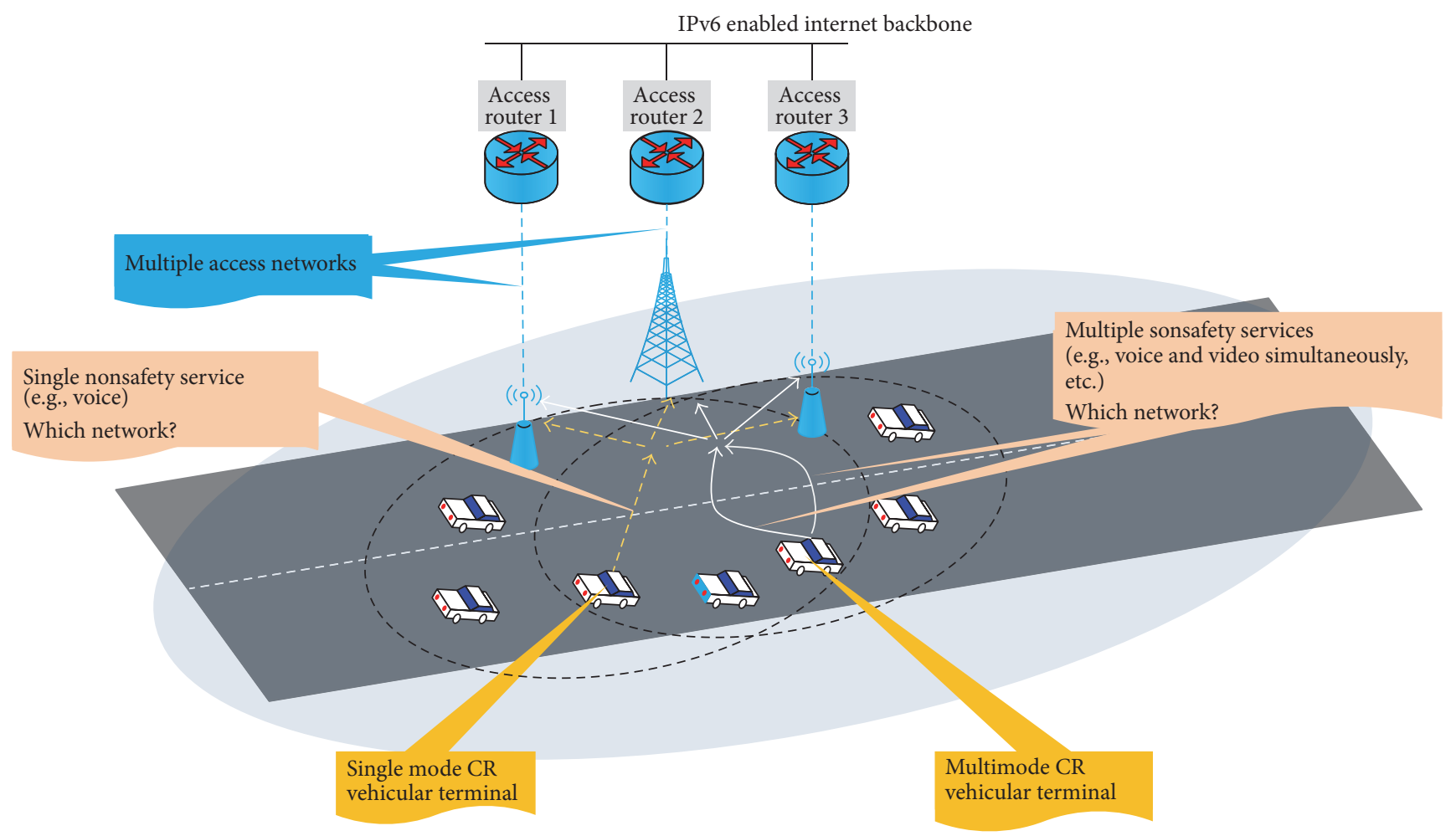

FIGURE 2: System model showing access network selection in single nonsafety service call/multiple nonsafety services call simultaneously in $\mathrm{CR}$ vehicular networks.

the safety service, reflex action is done for the spectrum handoff decision. The safety services always have high priority over the nonsafety services. There are four types of nonsafety services in vehicular networks such as voice, video, best effort, and background services. In this paper, CR vehicular nodes considered are multimode type in nature which has the capability to make call for two or more types of nonsafety services such as voice, video, and best effort simultaneously. The paper shows that proposed spectrum handoff scheme can be effectively implemented for spectrum handoff decision to select optimal network.

In this paper, we propose a spectrum handoff scheme for optimal network selection in NEMO based CR vehicular networks using MADM methods. The paper makes the following key research contributions:

(i) applicable for multimode CR vehicular nodes which have the capability to make call for two or more types of non-safety services simultaneously.

(ii) first approach performing spectrum handoff in NEMO based CR vehicular networks,

(iii) first approach performing spectrum handoff using MADM in NEMO based CR vehicular networks.

The rest of the paper is organized as follows. Section 2 presents the related works. In Section 3 and MADM methods are discussed. The proposed spectrum handoff scheme is presented in Section 4. The numerical results are presented and discussed in Section 5. Finally, the concluding remarks are given in Section 6.

\section{Related Works}

Recently, we have provided a comprehensive survey work on spectrum handoff schemes in CR networks [6]. At present, there is no literature available for spectrum handoff decision in NEMO based CR vehicular networks. However, there are only limited studies partially addressing this issue. The recent survey work by Singh et al. [11] advocates the use of NEMO protocol [17] for mobility management in CR vehicular networks, defined by the Internet Engineering Task Force (IETF).

Zhi-Jin et al. [18] propose spectrum handoff scheme using adaptive weights strategy considering $\mathrm{CR}$ information for CR networks. The least mean square algorithm is proposed and Simple Additive Weighting (SAW) method is used for the spectrum handoff decision. It is able to reduce the spectrum handoff time and effective data transmission time. The scheme is applicable for general CR networks that use SAW method only. The application of other MADM methods can further improve the scheme. Haldar et al. [19] propose a cross-layer architecture framework for network and channel selection in a heterogeneous cognitive wireless networks. In it, analytic hierarchy process method is used to classify the user applications and then prioritizing them based on the performance matrices. The proposed scheme outperforms the other compared schemes. But, its limitation is in terms of increased numbers of handoffs. Tumuluru et al. [20] have investigated dynamic spectrum access schemes considering the priority based spectrum handoff scheme under different CR networks architectures. CR unlicensed traffics are 
classified as high priority and low priority classes for the spectrum handoff decision. The application of MADM methods for traffic classification can further improve the presented scheme. Xian et al. [21] propose a novel grade-based spectrum handoff scheme for CR networks. The scheme is based on diversity of spectrum quality and traffic requirement of $\mathrm{CR}$ nodes. The scheme provides quality of service (QoS) guarantee for CR. But, it is applicable for general CR networks.

There is extensive research work available for general vehicular networks. In [22], a context aware based handoff scheme with multiple attributes for vehicular networks. The presented scheme is able to select the best network in vehicular networks. Prakash et al. [12] proposed unique vehicle assisted cross layer handoff based vertical handoff algorithm for VANEMO [vehicular ad hoc network (VANET) with network mobility] based on IPv6. This type of handoff scheme is applicable in VANET with NEMO. The main feature of the scheme is that it outperforms standard IETF NEMO basic support protocol with respect to handoff latency and packet loss during handoff period. Prakash et al. [23] also introduced another handoff scheme using NEMO for vehicular environment. The performance of the handoff mechanism is analyzed in multiple MR based mobile networks. Here, MRs cooperatively receive packets destined for each other and significantly reduce packet loss during handoff. Shen et al. [24] propose a dynamic mobility management framework for IPv6 based NEMO heterogeneous wireless networks which includes GRA method to improve decision making. Bakmaz et al. [25] have introduced the entropy method for weights estimation of multiple attributes considering/without considering user preferences. The weights are determined based on attributes entropy. The entropy method is more accurate and easy to apply. The Technique for Order of Preference by Similarity to Ideal Solution (TOPSIS) MADM method is for optimal network selection algorithm in wireless heterogeneous environment.

Our comprehensive survey work [6] on CR networks spectrum handoff schemes advocates the ample use of multiple attributes for spectrum handoff decision considering user preferences and application oriented based on dynamic network environment conditions. Recently, we have investigated a spectrum handoff scheme [16] for CR vehicular networks which takes the multiple attributes for the spectrum handoff decision considering nonsafety services. The scheme provides the spectrum handoff decision with wider and optimum choice with QoS considering nonsafety services. The investigated scheme is applicable for single mode CR vehicular nodes which have the capability for making single call of nonsafety services.

In brief, the prior works show that MADM methods are well applicable for handoff decision in next generation wireless networks. These methods provide well optimized results with reduced complexity problem in handoff decision. The efforts have been undertaken for the spectrum handoff in CR networks. Till now, there is no spectrum handoff scheme for optimal network selection for specific CR vehicular networks such as NEMO based CR vehicular networks. There is no spectrum handoff scheme considering multimode CR vehicular node having the capability to make call for two or more types of nonsafety services such as voice, video, and best effort simultaneously. This motivates us to work further to develop a new spectrum handoff scheme for multimode CR vehicular nodes having the capability for two or more nonsafety services simultaneously together for NEMO based CR vehicular networks.

\section{Multiple Attributes Decision Making}

The decision makers are often faced with several conflicting alternative. How do they evaluate trade-off when there are more than two alternatives to select the optimal alternative? MADM [26-29] is one of the best strategy to make decision for selecting an optimal alternative from a predetermined number of alternatives having multiple attributes. MADM methods specify how attribute values are processed in order to arrive at optimal choice. There are several MADM methods such as SAW [30, 31], GRA [13-15], TOPSIS [32-34], and cost based [16], which are extensively discussed in the literature. MADM methods can be applied for optimal network selection for the spectrum handoff decision in NEMO based CR vehicular networks. MADM methods are used for objective weights estimation as shown in Figure 3. Figure 3 shows different stages for weight estimation using MADM methods.

The summary of main notations used in paper is shown as follows:

$D$ : the multiple attributes matrix which contains multiple attributes w.r.t. alternatives,

$A_{i j}$ : the element of matrix, $D$ representing the value of attribute $i$ w.r.t. alternative $j$,

$\omega_{1}, \omega_{2}, \ldots, \Phi_{n}$ : the weights assigned to multiple attributes,

$r_{i j}$ : the multiple attributes normalized value,

$e_{i j}$ : the entropy of the multiple attributes with respect to alternative $j$,

$d$ : the deviation within each criterion with respect to the entropy,

$w$ : the weight selected according to the service or without service,

$U_{j}$ : the upper bound attribute value with respect to alternative $j$,

$I_{j}$ : the lower bound attribute value with respect to alternative $j$,

$\mathrm{GRC}_{i}$ : the GRC value for each alternative which is used for optimal network selection in GRA method,

$C_{i}$ : the cost value for each alternative which is used for optimal network selection in proposed cost based MADM method,

DR: data rate (Mbps),

PLR: packet loss ratio (per $10^{6}$ ),

TD: traffic density (\% user),

Dir: direction (degree),

PC: power consumption (w). 


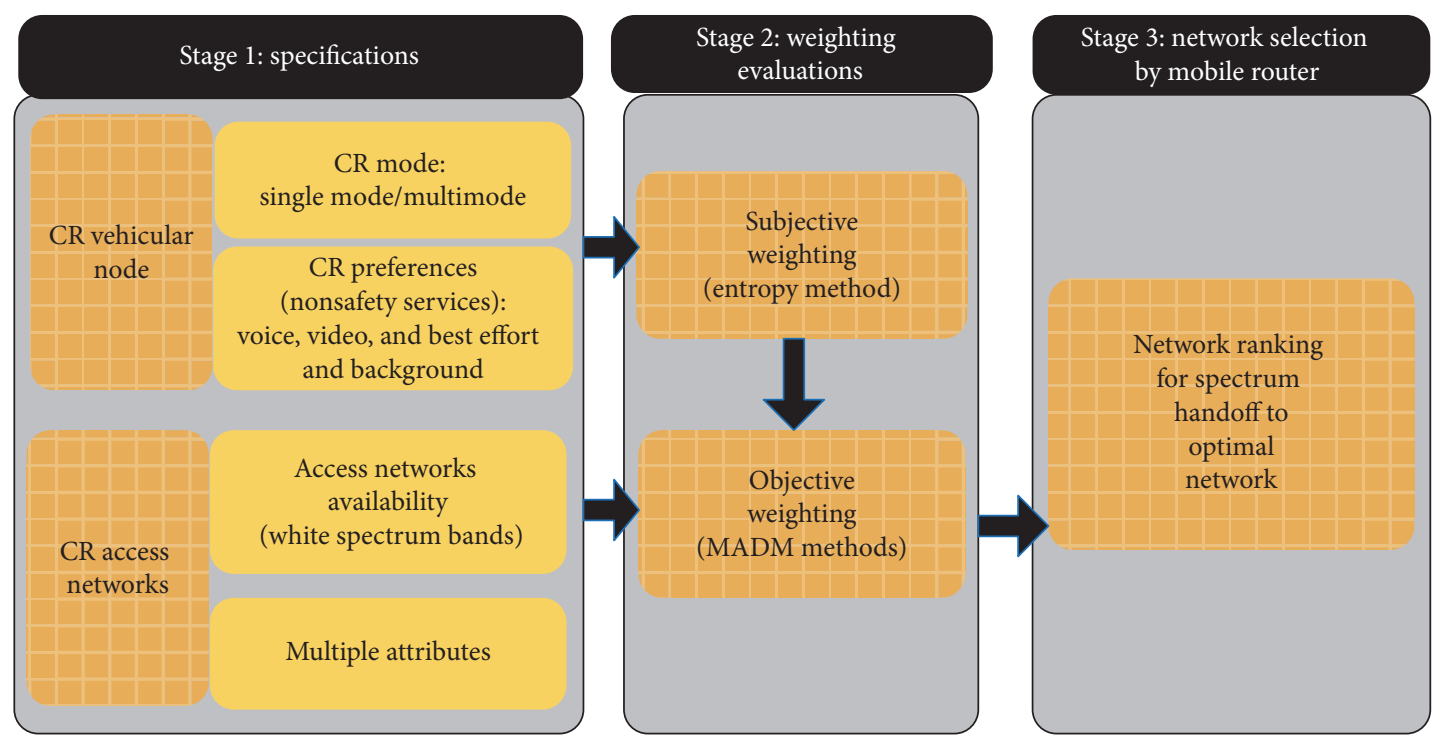

FIGURE 3: Weights estimation stages for network ranking for spectrum handoff decision.

In order to provide efficient spectrum handoff for optimal network selection in the presence of a number of decisions attributes, the multiple attributes matrix, $D$, is represented by

$D$

\begin{tabular}{|c|c|c|c|c|c|c|}
\hline \multicolumn{2}{|l|}{ Available } & \multicolumn{5}{|c|}{ Multiple Attributes } \\
\hline Alternatives & $\omega_{1}$ & $\omega_{2}$ & . & . & . . & $\omega_{n}$ \\
\hline$A_{1}$ & $A_{11}$ & $A_{12}$ & - & $\cdot$ & $\cdot \cdot$ & $A_{1 n}$ \\
\hline$A_{2}$ & $A_{21}$ & $A_{22}$ & . & . & . . & $A_{2 n}$ \\
\hline . & . & . & · & . & . . & . \\
\hline . & . & . & · & $A_{i j}$ & . & . \\
\hline . & . & . & . & $\cdot$ & . . & . \\
\hline . & . & . & . & . & . . & . \\
\hline$A_{m}$ & $A_{m 1}$ & $A_{m 2}$ & & . & . . & $A_{m n}$ \\
\hline
\end{tabular}

where $A_{i j}$ represents the element of matrix. It represents the value of attribute $i$ with respect to alternative $j . \omega_{1}, \omega_{2}, \ldots, \omega_{n}$ are the weights assigned to multiple attributes. The alternatives are $A_{1}, A_{2}, \ldots, A_{m}$ of different access networks available for spectrum handoff in NEMO based CR vehicular networks.

3.1. Subjective Weights Estimation by Entropy Method. The entropy method [25] is used to generate the subjective weights for multiple attributes. The weights are determined based on attributes entropy. The entropy method is accurate and easy to apply. The main characteristic of the method is that the weights are computed considering/without considering user preferences. It consists of the following steps.

(1) The Multiple Attributes Transformation. The multiple attributes are transformed into two categories. One category is the benefit attributes which means maximum the best. The other category is cost attributes which means minimum the best.
Hence, the nature of the cost attributes like delay, PLR, price per unit, jitter, traffic load, power consumption, and direction change from minimum to maximum using

$$
A_{i j}^{*}=\frac{1}{A_{i j}} \quad \text { where } i \in\{1,2, \ldots, m\}, j \in\{1,2, \ldots, n\} .
$$

The nature of benefit attributes like data rate does not change for the normalization.

(2) The Multiple Attributes Normalized Value Estimation. It is necessary to transform the model in such a way that the attributes value of different access networks must be maximized.

If nature of the attributes does not change, the normalized value, $r_{i j}$, is computed as

$$
r_{i j}=\frac{A_{i j}}{\sqrt{\sum_{i=1}^{m} A_{i j}^{2}}}
$$

where $i \in\{1,2, \ldots, m\}, j \in\{1,2, \ldots, n\}$.

If nature of the attributes changes, $r_{i j}$ is computed as

$$
\begin{aligned}
r_{i j}=\frac{A_{i j}^{*}}{\sqrt{\sum_{i=1}^{m}\left(A_{i j}^{*}\right)^{2}}} & \quad \text { where } i \in\{1,2, \ldots, m\}, j \in\{1,2, \ldots, n\} .
\end{aligned}
$$

(3) Entropy Estimation. The entropy of the multiple attributes is calculated as follows:

$$
e_{i j}=\left[\frac{-1}{\ln (n)}\right] \times \sum_{i=1}^{m}\left[r_{i j} \ln \left(r_{i j}\right)\right]
$$

where $i \in\{1,2, \ldots, m\}, j \in\{1,2, \ldots, n\}$. 
TABLE 1: Attributes priority values.

\begin{tabular}{lcc}
\hline $\begin{array}{l}\text { Attributes } \\
\text { priority }\end{array}$ & Priority level & Priority value \\
\hline Highest & 6 & 0.65 \\
Very high & 5 & 0.40 \\
High & 4 & 0.30 \\
Medium & 3 & 0.20 \\
Low & 2 & $0.01-0.05$ \\
Equal & 1 & 1 \\
\hline
\end{tabular}

(4) Deviation Calculation. The deviation within each criterion is as follows

$$
d_{i j}=1-e_{i j}
$$

(5) Weight Coefficients Computation. If user equally prefers all the attributes, there is no user preference for the weights computation. The weight coefficients are computed as follows:

$$
w_{i j}=\frac{d_{i j}}{\sum_{j=1}^{n} d_{i j}}
$$

where $i \in\{1,2, \ldots, m\}, j \in\{1,2, \ldots, n\}$.

Subjective Weights. The subjective weights are computed using user preferences. If CR vehicular nodes determines the subjective weights,

$$
w_{i j}=\frac{d_{i j} \omega_{i j}}{\sum_{j=1}^{n} d_{i j}},
$$

where $w_{i j}$ is the proffered weight called weights selected according to the service, and $\omega_{i 1}+\omega_{i 2}+\omega_{i 3}+\omega_{i 4}+\cdots=1$, where $\omega_{i 1}, \omega_{i 2}, \omega_{i 3}, \omega_{i 4}$ are the normalized proffered weights assigned to multiple attributes whose values are dependent on CR services using Table 1 . Table 1 shows multiple attributes priority levels where crisp and linguistic terms are converted into fuzzy number. Each priority levels have priority values which are used as proffered weights. for example, the delay should be minimum for voice service. Hence, the delay attribute has highest priority level in case of voice. Therefore, proffered weight for delay in voice service is 0.65 . If the user preferences are not considered, then the multiple attributes have equal priority which means no priority for the multiple attributes. In this case, the priority value is estimated by $1 /$ (number of multiple attributes).

Hence, user preferences are considered for subjective weights estimation for nonsafety services. The subjective weights for $\mathrm{CR}$ vehicular nodes which have the capability to make call for two or more types of nonsafety services simultaneously. These subjective weights are determined by using (8). The different combinations of two or more types of nonsafety services are (i) voice and video services, (ii) voice and best effort services, (iii) video and best effort services, and (iv) voice, video, and best effort service, as there is no need of preference for the background service. Hence, the weights are computed using (7).
3.2. Grey Relational Analysis Method. Grey system theory has introduced GRA method [13-15]. It is used to solve the uncertainty problems under discrete data sequence with partial information. In grey system theory, the degree of information is used to define a system. If the information of the system is fully known, it is known as white system. If the system is unknown, it is known as black system. A system with the partial information is known as a grey system. One of the reference sequences is called ideal solution. The grey relation between reference sequence and other sequence is calculated using Grey Relational Coefficient (GRC). It consists of the following steps.

(1) Classification of Multiple Networks Attributes. The attributes like delay, PLR, price per unit, jitter, traffic load, power consumption, and direction are categorized as smaller the best category, called cost attributes. The data rate should be high. Hence, it is catergorized as larger, the better category. It is called benefit attribute. These attributes are classified using step 1 of entropy method.

(2) Determine Upper Bound and Lower Bound Attributes. The multiple attributes matrix, $D$, consists of $\mathrm{m}$ networks such as $A_{1}, A_{2}, \ldots, A_{m}$. The upper bound $U_{j}$ is determined as $\max \left(A_{1}(j), A_{2}(j), \ldots, A_{m}(j)\right)$ and the lower bound $I_{j}$ is determined as $\min \left(A_{1}(j), A_{2}(j), \ldots, A_{m}(j)\right)$, where $j=$ $1,2, \ldots, n$.

(3) Max-Min Normalization. In case the normalization of attributes is smaller, the best category is computed as

$$
A_{i}^{*}(j)=\frac{U_{j}-A_{i}(j)}{U_{j}-I_{j}}
$$

and in case it is larger the better category is computed as

$$
A_{i}^{*}(j)=\frac{A_{i}(j)-U_{j}}{U_{j}-I_{j}} .
$$

Hence, the normalized matrix, $D_{\text {Norm }}$, can be represented as

$$
D_{\text {Norm }}=\left[\begin{array}{cccc}
A_{1}^{*}(1) & A_{1}^{*}(2) & \cdots & A_{1}^{*}(n) \\
A_{2}^{*}(1) & A_{2}^{*}(n) & \cdots & A_{2}^{*}(n) \\
\vdots & \vdots & \cdots & \vdots \\
A_{m}^{*}(1) & A_{m}^{*}(2) & \cdots & A_{m}^{*}(n)
\end{array}\right] .
$$

(4) Computation of GRC Values. The values of GRC are calculated by the following equation array as follows:

$$
\mathrm{GRC}_{i}=\frac{1}{\left[1+\sum_{j=1}^{n} w_{i j}\left|A_{j}^{*}(n)-1\right|\right]},
$$

where $w_{i j}$ is the subjective weight of each attribute and $i$ ( $1 \leq$ $i \leq m)$.

(5) Networks Ranking. The best network is according to the descending order of GRC values. Hence, network with the highest GRC value is selected as optimal network for the spectrum decision in NEMO based CR vehicular networks. 
3.3. Cost Based MADM Method. The cost based method [16] is proposed to formulate as MADM method. It evaluates the cost of multiple attributes of different access networks for spectrum handoff decision to select optimal network in NEMO based CR vehicular networks. The advantage of this method is that the multiple attributes are used directly for the estimation of cost value. Hence, it is simple and easy to implement. It consists of the following steps.

(1) Multiple Attributes Transformation. All attributes value of different access networks are transformed to its maximum value. The nature of the cost attributes like delay, PLR, price per unit, jitter, traffic load, power consumption, and direction changes from minimum to maximum as follows:

$$
A_{i j}^{*}=\frac{1}{A_{i j}} \quad \text { where } i \in\{1,2, \ldots, m\}, j \in\{1,2, \ldots, n\} .
$$

The nature of benefit attribute like data rate attribute does not change for the normalization.

(2) Vector Normalization of Attributes. If the nature of the attributes does not change, the normalized value, $r_{i j}$ is computed as

$$
r_{i j}=\frac{A_{i j}}{\sqrt{\sum_{i=1}^{m} A_{i j}^{2}}} .
$$

If nature of the attributes changes, $r_{i j}$ is computed as

$$
r_{i j}^{*}=\frac{A_{i j}^{*}}{\sqrt{\sum_{i=1}^{m}\left(A_{i j}^{*}\right)^{2}}}
$$

where $i \in\{1,2, \ldots, m\}, j \in\{1,2, \ldots, n\}$.

(3) Cost Value Computation. The cost value is computed as follows:

$$
C_{i}=\sum_{j=1}^{n} \frac{w_{i j}\left(A_{i j}\right)}{r_{i j}},
$$

where $i \in\{1,2, \ldots, m\}, j \in\{1,2, \ldots, n\}$. The value of $w_{i j}, A_{i j}$, and $r_{i j}$ are determined from (8), (14), and (15), respectively.

(4) Networks Ranking. The best network is according to the descending order of cost values. Hence, network with the highest cost value is selected as an optimal network for the spectrum decision in CR vehicular networks.

\section{Proposed Spectrum Handoff Scheme}

In this section, the proposed spectrum handoff scheme for optimal network selection in NEMO based CR vehicular networks is presented and discussed. The information from NEMO based CR vehicular networks having white spectrum bands is available in terms of the multiple attributes matrix $\left(D_{i j}\right)$.

The proposed spectrum handoff scheme is shown in Figure 4. It consists of the following steps.
(1) Initially, the multiple attributes matrix $\left(D_{i j}\right)$, called decision matrix, is formed by getting input from NEMO based CR vehicular networks as

$D_{i j}=$ (Data rate, delay, packet loss ratio, jitter, price,

traffic density, direction, power consumption),

where $i$ represents the access network, $i=(1,2, \ldots$, $m)$, and $j$ represents its multiple attributes, $j=$ $(1,2, \ldots, n)$.

(2) The multiple attributes are transformed into benefit and cost attributes. The benefit attributes mean maximum the best like data rate. The cost attribute means minimum the best like delay. These transformations are based on the entropy, GRA, and cost based MADM methods.

(3) The multiple attributes normalized values are obtained using vector normalization and max-min normalization. The max-min normalization values are used in GRA method and vector normalization values are used in cost based method.

(4) The subjective weights are computed by the entropy method with or without considering CR vehicular node preferences. The CR vehicular node has the capability to make single as well as group of two or more calls simultaneously for different nonsafety services.

(5) The optimal network is selected for the spectrum handoff using GRA and cost based methods. Using GRA method, the optimal network is selected according to the descending order of weighted average values and GRC values, respectively. Similarly, cost based method select optimal network according to the highest cost value.

\section{Results and Discussion}

In this section, we apply GRA and cost based method for the proposed scheme. For the evaluation purpose, a NEMO based CR vehicular networks is considered which integrates WiMax, WLAN, LTE, and satellite networks. Table 2 shows the multiple attributes values of the considered networks at the time of the network selection whose information is stored with the wireless service providers. Figure 5(a) shows graphical representation of the weight generated by the entropy method without considering CR vehicular node preferences whose sum is 1. Figure 5(b) presents the network selection ranking without considering $\mathrm{CR}$ vehicular node preferences. Figure 5(b) shows that GRA method selects WLAN and cost based method selects LTE as an optimal network for spectrum handoff for nonsafety services in NEMO based CR vehicular networks. This is a static methodology to select the optimal network for spectrum handoff. However, this static methodology does not provide the optimal network for the spectrum handoff decision. Table 2 shows that WLAN is not an optimal network for voice service in comparison with WiMax, LTE, and satellite networks. The delay is not minimum in WLAN which is the dominant attribute in case 


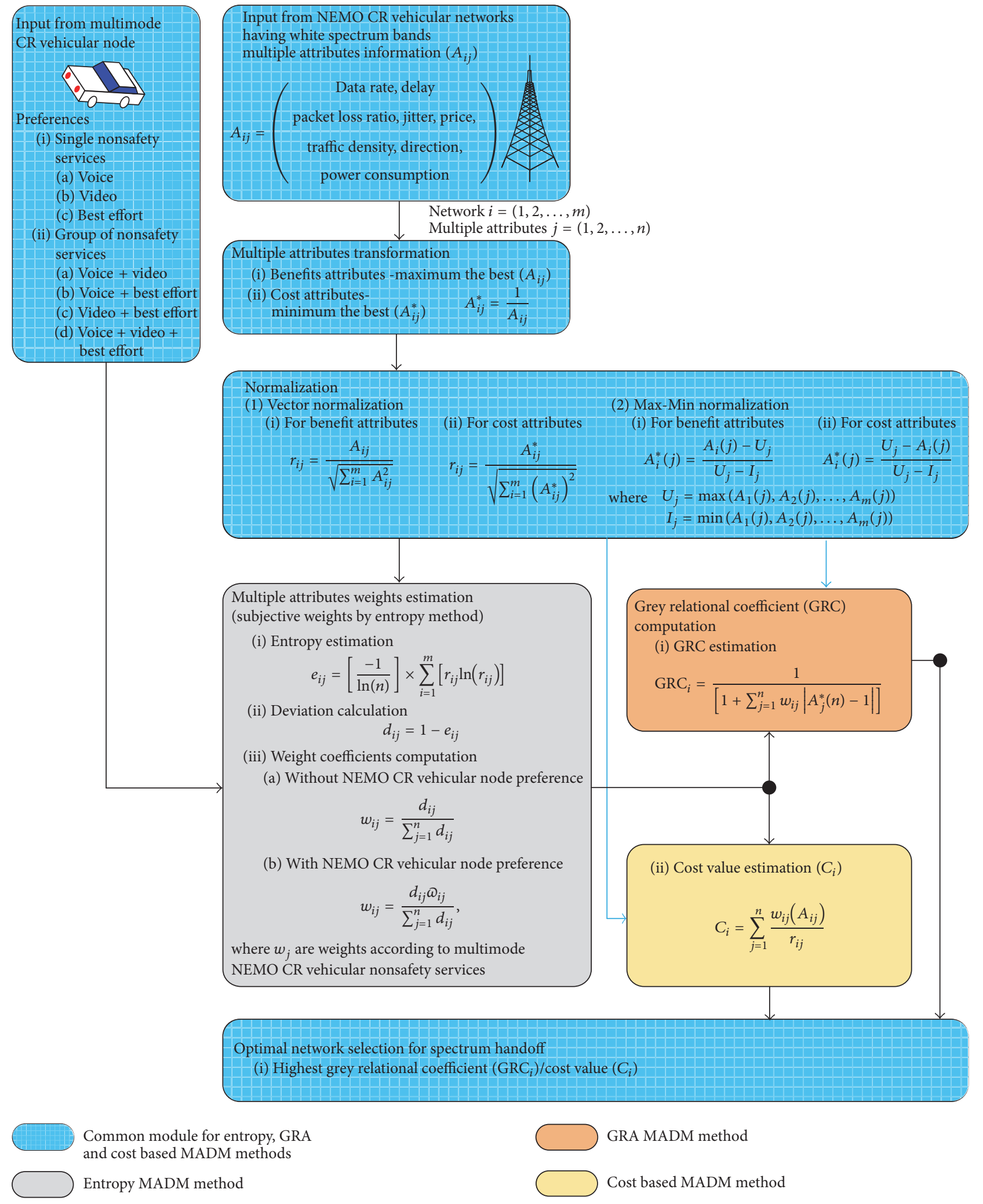

FIGURE 4: Spectrum handoff scheme using multiple attributes group decision making method for optimal network selection in NEMO based CR vehicular networks. 
TABLE 2: Multiple attributes matrix of different access networks.

\begin{tabular}{|c|c|c|c|c|c|c|c|c|}
\hline \multirow{2}{*}{ Access networks } & \multicolumn{8}{|c|}{ Multiple attributes } \\
\hline & DR (Mbps) & Delay (ms) & $\operatorname{PLR}\left(\operatorname{per} 10^{6}\right)$ & Jitter (ms) & Price (per unit) & TD (\% user) & Dir (degree) & $\mathrm{PC}(\mathrm{w})$ \\
\hline Network 1: WiMax & 35 & 70 & 45 & 6 & 4 & 40 & 15 & 5 \\
\hline Network 2: WLAN & 11 & 60 & 30 & 10 & 3 & 12 & 60 & 2 \\
\hline Network 3: LTE & 70 & 15 & 25 & 9 & 10 & 90 & 40 & 3 \\
\hline Network 4: satellite & 2 & 200 & 80 & 20 & 13 & 20 & 5 & 4 \\
\hline
\end{tabular}

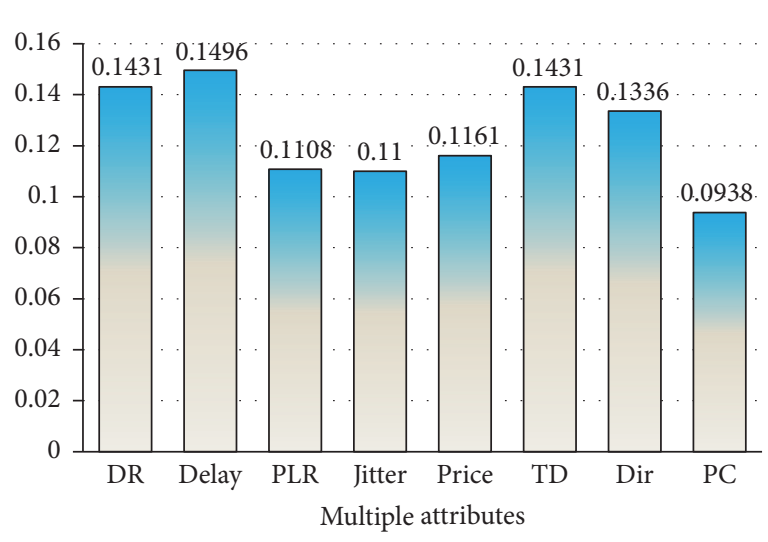

$\square$ Attributes weights

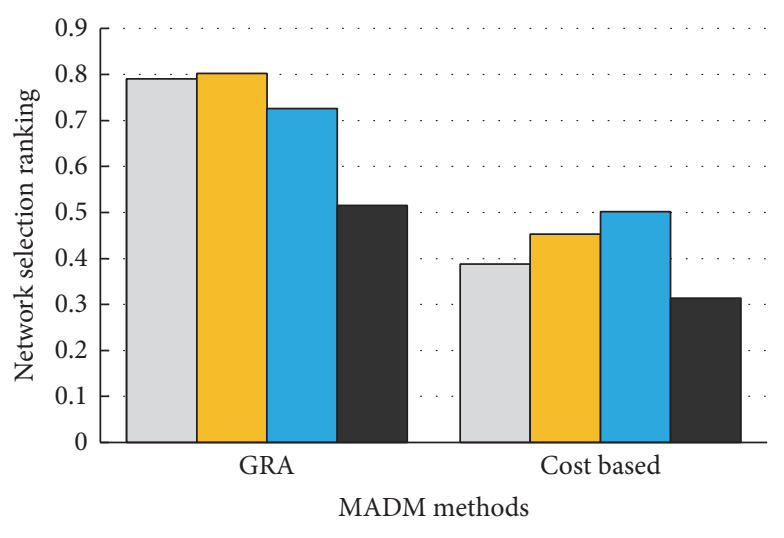

$\square$ Network 1: WiMax $\square$ Network 2: WLAN $\square$ Network 3: LTE

- Network 4: satellite

(a)

FIGURE 5: (a) Representation of multiple weights generated by the entropy method without considering CR vehicular node preferences and (b) network selection ranking for spectrum handoff without considering CR vehicular node preferences.

of voice service. Hence, CR vehicular node preferences need to be considered for optimal network selection. Therefore, all the following subsection has considered CR vehicular node preferences for optimal network selection for spectrum handoff decision.

\subsection{Performance Evaluation of the Proposed Scheme for Inter-} network Spectrum Handoff. This subsection evaluates the performance of the proposed spectrum handoff scheme for CR vehicular node which has the capability to make single and multiple nonsafety services simultaneously.

\subsubsection{Performance Evaluation of the Internetwork Spectrum Handoff Scheme for Single Nonsafety Service}

Case 1 (voice service). In this case, $\mathrm{CR}$ vehicular requirement is voice service. For the voice service, the minimum delay is desirable. It is considered as a dominant attribute. Therefore, high priority level should be considered for delay as compared to other attributes as shown in Table 3. Figure 6 shows weights selected by the entropy method considering the CR vehicular node preference of single nonsafety service. Figure 6 shows that the voice has high weightage compared to other attributes. In Figure 7, Scenario la shows LTE network has highest network selection ranking computed by GRA and cost based methods. Hence, LTE is an optimal network for the spectrum decision. It can be verified from Table 2 that the

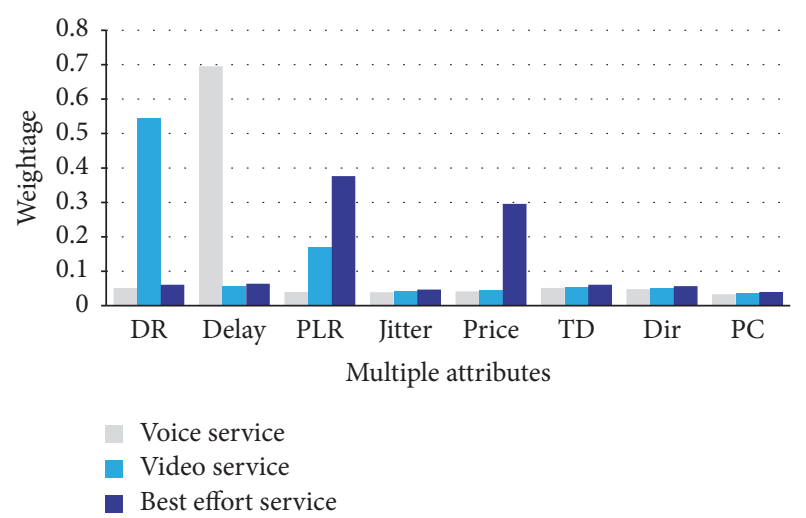

FIGURE 6: Weight selected by entropy method for internetwork spectrum handoff for single nonsafety services admitted by CR vehicular node.

delay in LTE network is minimum which is desirable for the voice.

Case 2 (video service). For video service, data rate and PLR are the dominant attributes compared with other attributes as shown in Table 3. Figure 6 shows the weights selected by entropy method considering CR vehicular preference. In Figure 7, Scenario $1 \mathrm{~b}$ shows that both GRA and cost based method select LTE as an optimal network for spectrum 
TABLE 3: Priority level for internetwork spectrum handoff for single non-safety service admitted by CR vehicular node.

\begin{tabular}{lccccccc}
\hline \multirow{2}{*}{ Nonsafety services } & \multicolumn{9}{c}{ Priority level } & Jitter & Price & TD & Dir & PC \\
\hline Voice service & DR & Delay & PLR & 2 & 2 & 2 & 2 \\
Video service & 2 & 6 & 3 & 2 & 2 & 2 & 2 \\
Best effort service & 2 & 2 & 5 & 2 & 4 & 2 & 2 \\
Background service & 1 & 1 & 1 & 1 & 1 & 1 & 1 \\
\hline
\end{tabular}
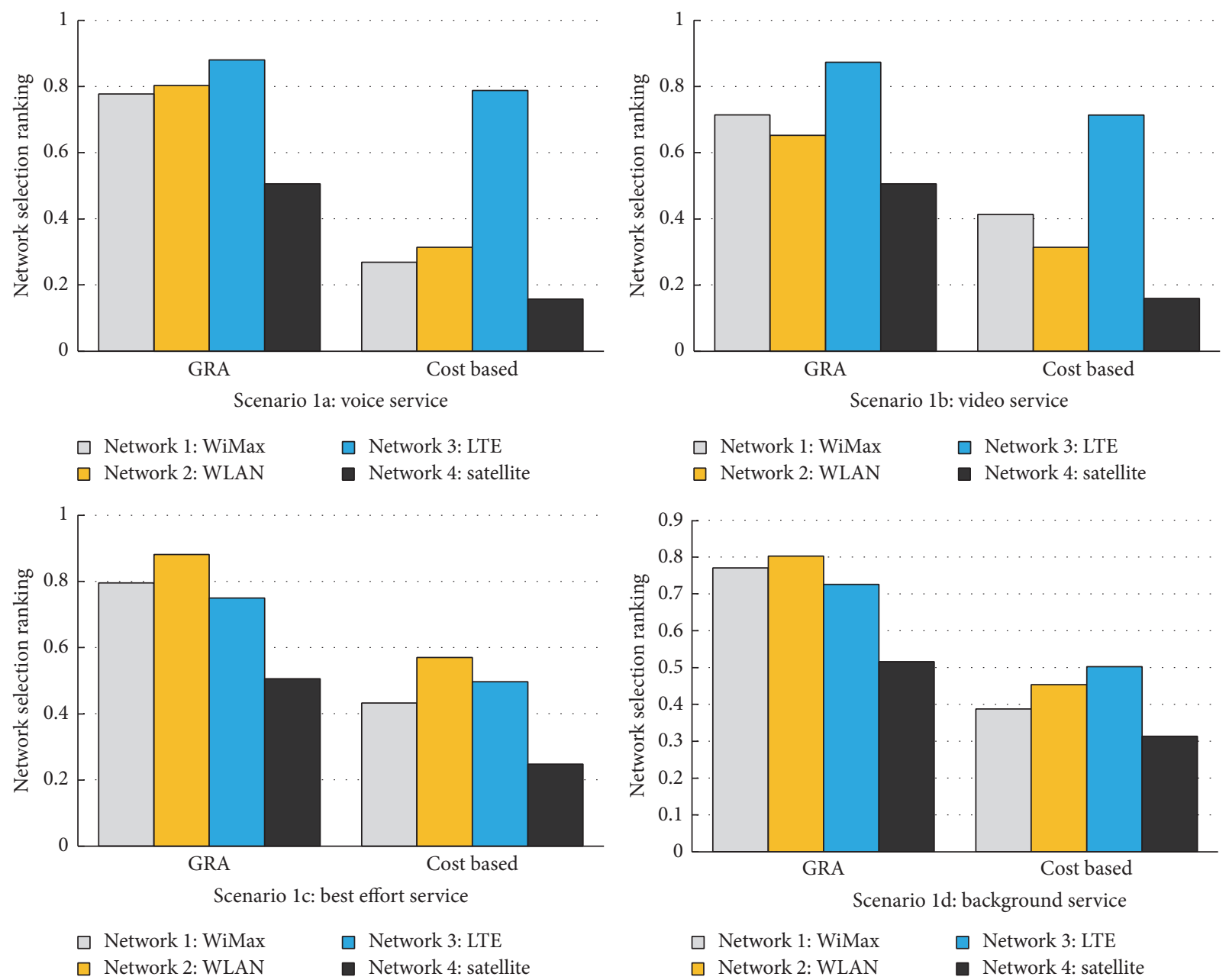

FIGURE 7: Optimal network selection for spectrum handoff for internetwork spectrum handoff for single nonsafety service admitted by CR vehicular node. Scenario la: voice service; Scenario 1b: video service; Scenario 1c: best effort service; Scenario 1d: background service.

handoff for video service. It can be verified from Table 2 that LTE has minimum data rate and PLR as compared to other available access networks which is desirable.

Case 3 (best effort service). For the best effort service, the dominant attributes are PLR and price as shown in Table 3. Figure 6 shows the weightage for the attributes considering CR vehicular node preference. In Figure 7, Scenario 1c shows that both GRA and cost based methods select WLAN as an optimal network for spectrum handoff which is desirable. It can be verified from Table 2 that WLAN has minimum PLR and price as compared to the other available access networks.
Case 4 (background service). CR vehicular node does have any preferences for the background service. Hence, there is no need of using CR vehicular node preferences for this service as shown in Table 3. All the attributes have equal priority level. GRA and cost based method select different access network for the spectrum handoff decision as shown in Figure 7, Scenario 1d. GRA method selects LTE and cost based method selects WiMax network as an optimal network. As the background service does not have any preference, so LTE/WiMax method can be used as an optimal network for all cases in NEMO based CR vehicular network. Therefore, background service will not be discussed in the rest of section. 
TABLE 4: Optimal networks selection for spectrum handoff for internetwork spectrum handoff for single nonsafety service admitted by CR vehicular node.

\begin{tabular}{|c|c|c|c|c|c|}
\hline Case & Scenario & Nonsafety service & MADM method & Optimal network selection ranking & Optimal network selection \\
\hline \multirow{2}{*}{1} & \multirow{2}{*}{ la } & \multirow{2}{*}{ Voice service } & GRA method & 0.8807 & LTE \\
\hline & & & Cost based method & 0.7881 & LTE \\
\hline \multirow{2}{*}{2} & \multirow{2}{*}{$1 b$} & \multirow{2}{*}{ Video service } & GRA method & 0.8738 & LTE \\
\hline & & & Cost based method & 0.7143 & LTE \\
\hline \multirow{2}{*}{3} & \multirow{2}{*}{$1 \mathrm{c}$} & \multirow{2}{*}{ Best effort service } & GRA method & 0.8813 & WLAN \\
\hline & & & Cost based method & 0.5703 & WLAN \\
\hline \multirow{2}{*}{4} & \multirow{2}{*}{$1 \mathrm{~d}$} & \multirow{2}{*}{ Background service } & GRA method & 0.8021 & LTE \\
\hline & & & Cost based method & 0.5201 & WiMax \\
\hline
\end{tabular}

TABLE 5: Priority level for triple play services for internetwork spectrum handoff for group of nonsafety services admitted by CR vehicular node.

\begin{tabular}{|c|c|c|c|c|c|c|c|c|}
\hline \multirow{2}{*}{ Non-safety services } & \multicolumn{8}{|c|}{ Priority level } \\
\hline & $\mathrm{DR}$ & Delay & PLR & Jitter & Price & $\mathrm{TD}$ & Dir & PC \\
\hline Voice + video service & 3 & 5 & 3 & 2 & 2 & 2 & 2 & 2 \\
\hline Voice + best effort service & 2 & 5 & 4 & 2 & 3 & 2 & 2 & 2 \\
\hline Video + best effort service & 3 & 2 & 3 & 2 & 5 & 2 & 2 & 2 \\
\hline Voice + video + best effort service & 3 & 5 & 2 & 2 & 3 & 2 & 2 & 2 \\
\hline
\end{tabular}

Table 4 shows the summary of optimal network selected for internetwork spectrum handoff scheme for single nonsafety service admitted by CR vehicular node.

\subsubsection{Performance Evaluation of the Internetwork Spectrum} Handoff Scheme for Group of Nonsafety Services. In this subsection, the performance of the proposed spectrum handoff scheme is evaluated for group of the nonsafety services admitted by $\mathrm{CR}$ vehicular node. The CR vehicular node has the capability to make group of call for two or more type of nonsafety services simultaneously. The multiple services selection will impact the optimal network selection for the spectrum handoff as there is need to take care of two or more services simultaneously.

Case 1 (voice + video service). In this case, $\mathrm{CR}$ vehicular node requirement is voice and video service simultaneously to make group of call. The dominant attribute for voice service is delay whereas video service has data rate and PLR as the dominant attributes. Hence, the dominant attributes for this case are delay, data rate, and PLR. Table 5 shows the attributes priority level and Figure 8 shows the corresponding weightage considering $\mathrm{CR}$ vehicular node requirement. In Figure 9, Scenario 2a shows both GRA and cost based methods select LTE as an optimal network for voice and video service call simultaneously. It can be verified from Table 2 that LTE has minimum delay and PLR as compared to other available access networks. Similarly, the data rate of LTE is maximum as compared to other available access network which is desirable.

Case 2 (voice + best effort service). In this case, CR vehicular node requirement is voice and best effort service simultaneously to make group of call. The dominant attributes

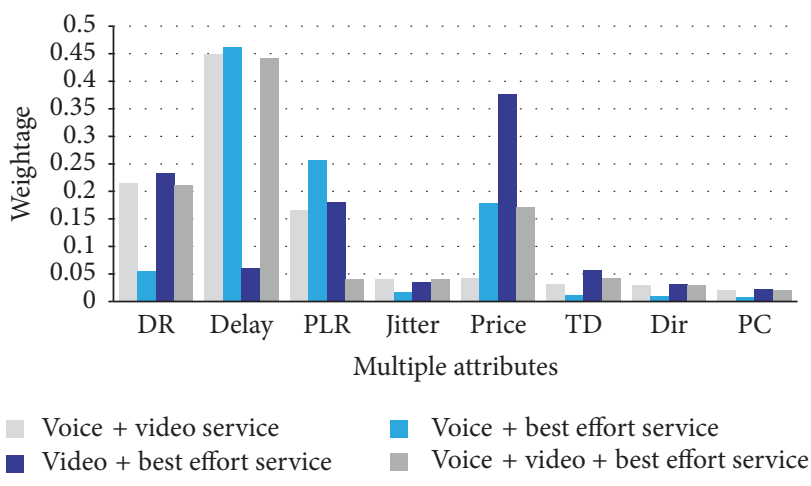

FIGURE 8: Weight selected by entropy method for internetwork spectrum handoff for group of nonsafety services admitted by CR vehicular node.

for this case are delay, PLR, and price. Table 5 shows the attributes priority level and Figure 8 shows the corresponding weightage considering $\mathrm{CR}$ vehicular node requirement. In Figure 9, Scenario 2b shows both GRA and cost based methods select LTE as an optimal network for voice and best effort service call simultaneously. It can be verified from Table 2 that LTE has minimum delay and PLR as compared to other available access networks which are desirable.

Case 3 (video + best effort service). In this case, $\mathrm{CR}$ vehicular node requirement is video and best effort service simultaneously to make group of call. The dominant attributes for this case are data rate, PLR, and price. Table 5 shows the attributes priority level and Figure 8 shows the corresponding weightage considering $\mathrm{CR}$ vehicular node requirement. In Figure 9, Scenario 2c shows both GRA and cost based methods select WLAN as optimal network for voice and 


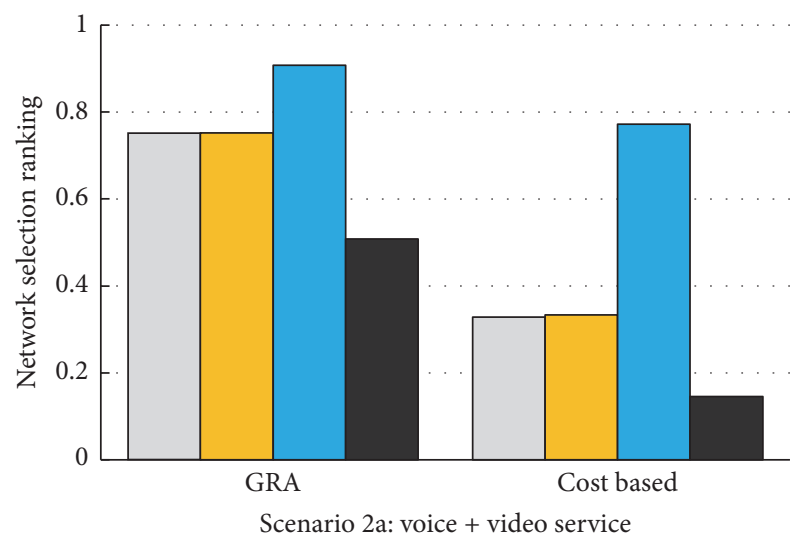

$\square$ Network 1: WiMax

$\square$ Network 2: WLAN

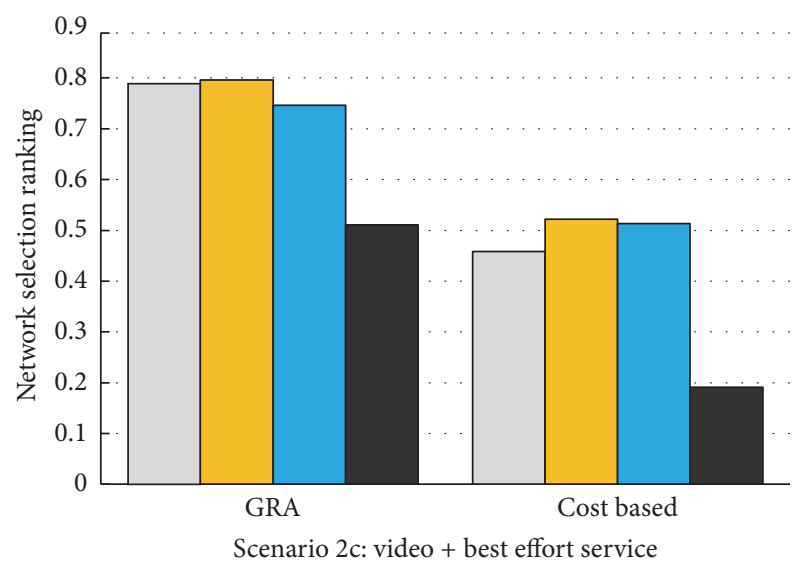

$\square$ Network 1: WiMax

$\square$ Network 2: WLAN $\square$ Network 3: LTE

- Network 4: satellite

$$
\begin{aligned}
& \square \text { Network 3: LTE } \\
& \square \text { Network 4: satellite }
\end{aligned}
$$

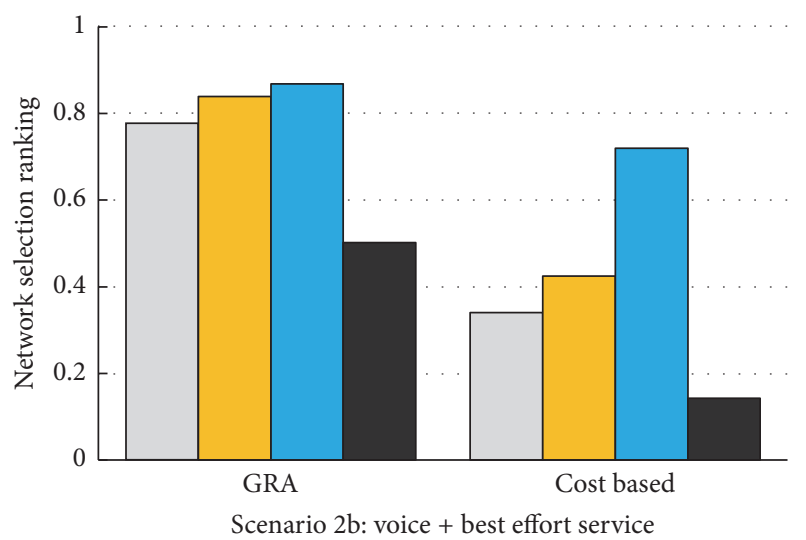
$\square$ Network 1: WiMax
$\square$ Network 3: LTE
$\square$ Network 2: WLAN
- Network 4: satellite

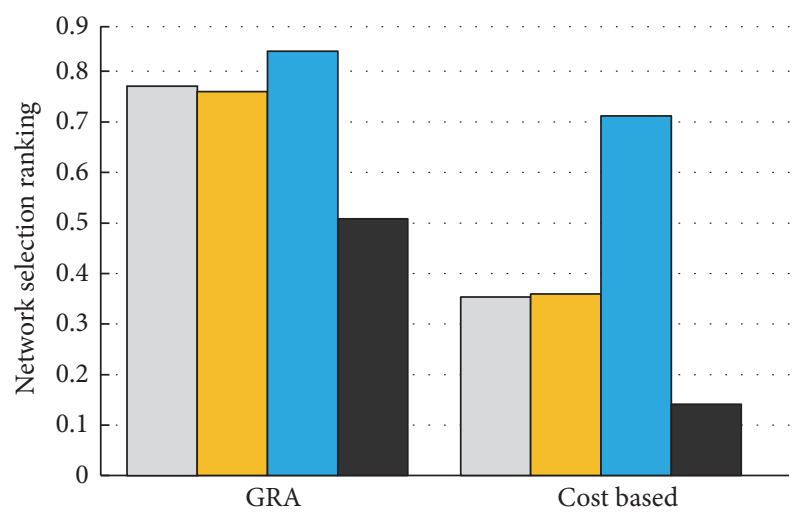

Scenario $2 \mathrm{~d}$ : voice + video + best effort service

$$
\begin{aligned}
& \square \text { Network 1: WiMax } \\
& \square \text { Network 2: Wi-Fi }
\end{aligned}
$$

FIGURE 9: Optimal network selection for spectrum handoff for internetwork spectrum handoff group of nonsafety services admitted by CR vehicular node. Scenario 2a: voice + video service; Scenario 2b: voice + best effort service; Scenario 2c: video + best effort service; Scenario 2d: voice + video + best effort service.

best effort service call simultaneously. It can be verified from Table 2 that WLAN has minimum price as compared to other available access networks which is desirable.

Case 4 (voice + video + best effort service). In this case, CR vehicular node requirement is voice, video, and best effort service simultaneously to make group of call. The dominant attributes considered for this case are delay, data rate, and price. Table 5 shows the attributes priority level and Figure 8 shows the corresponding weightage considering CR vehicular node requirement. In Figure 9, Scenario 2d shows both GRA and cost based methods select LTE as optimal network for voice, video, and best effort service call simultaneously. It can be verified from Table 2 that LTE has minimum delay and maximum data rate as compared to other available access networks which are desirable.

5.2. Impact of Velocity of Mobile Network on the Optimal Network Selection for the Internetwork Spectrum Handoff for Group of Nonsafety Services. In this case, the impact of velocity of mobile network on optimal network selection for internetwork spectrum handoff for group of nonsafety services admitted by $\mathrm{CR}$ vehicular node is evaluated. The cost based MADM method is used. When velocity of mobile network increases, the change in the direction of movement of mobile network/CR vehicular node is very less for a short period of time. Hence, the attribute priority level for the direction attribute also increases to select the network existing in the same direction. Table 2 shows that the value of the direction attribute is minimum for satellite network compared with other access networks. It means that satellite network exists in the direction of the movement of mobile network/CR vehicular network node.

Case 1 (voice + video service). In Figure 9, Scenario 2a and Table 6 show LTE is the optimal network for spectrum handoff for voice and video group of nonsafety service. In Figure 10, Scenario 3a shows that, with the increases in mobile network velocity, the network selection ranking decreases for LTE network and increases for satellite network. Hence, there is spectrum handoff from LTE to satellite network with the increase in mobile network velocity. 
TABLE 6: Optimal networks selection for spectrum handoff for internetwork spectrum handoff for single nonsafety service admitted by CR vehicular node.

\begin{tabular}{|c|c|c|c|c|c|}
\hline Case & Scenario & Nonsafety service & MADM method & Optimal network selection ranking & Optimal network selection \\
\hline \multirow{2}{*}{1} & \multirow{2}{*}{$2 \mathrm{a}$} & \multirow{2}{*}{ Voice + video service } & GRA method & 0.9079 & LTE \\
\hline & & & Cost based method & 0.7719 & LTE \\
\hline \multirow{2}{*}{2} & \multirow{2}{*}{$2 b$} & \multirow{2}{*}{ Voice + best effort service } & GRA method & 0.8688 & LTE \\
\hline & & & Cost based method & 0.7200 & LTE \\
\hline \multirow{2}{*}{3} & \multirow{2}{*}{$2 c$} & \multirow{2}{*}{ Video + best effort service } & GRA method & 0.7961 & WLAN \\
\hline & & & Cost based method & 0.5221 & WLAN \\
\hline \multirow{2}{*}{4} & \multirow{2}{*}{$2 \mathrm{~d}$} & \multirow{2}{*}{ Voice + video + best effort service } & GRA method & 0.8396 & LTE \\
\hline & & & Cost based method & 0.7122 & LTE \\
\hline
\end{tabular}
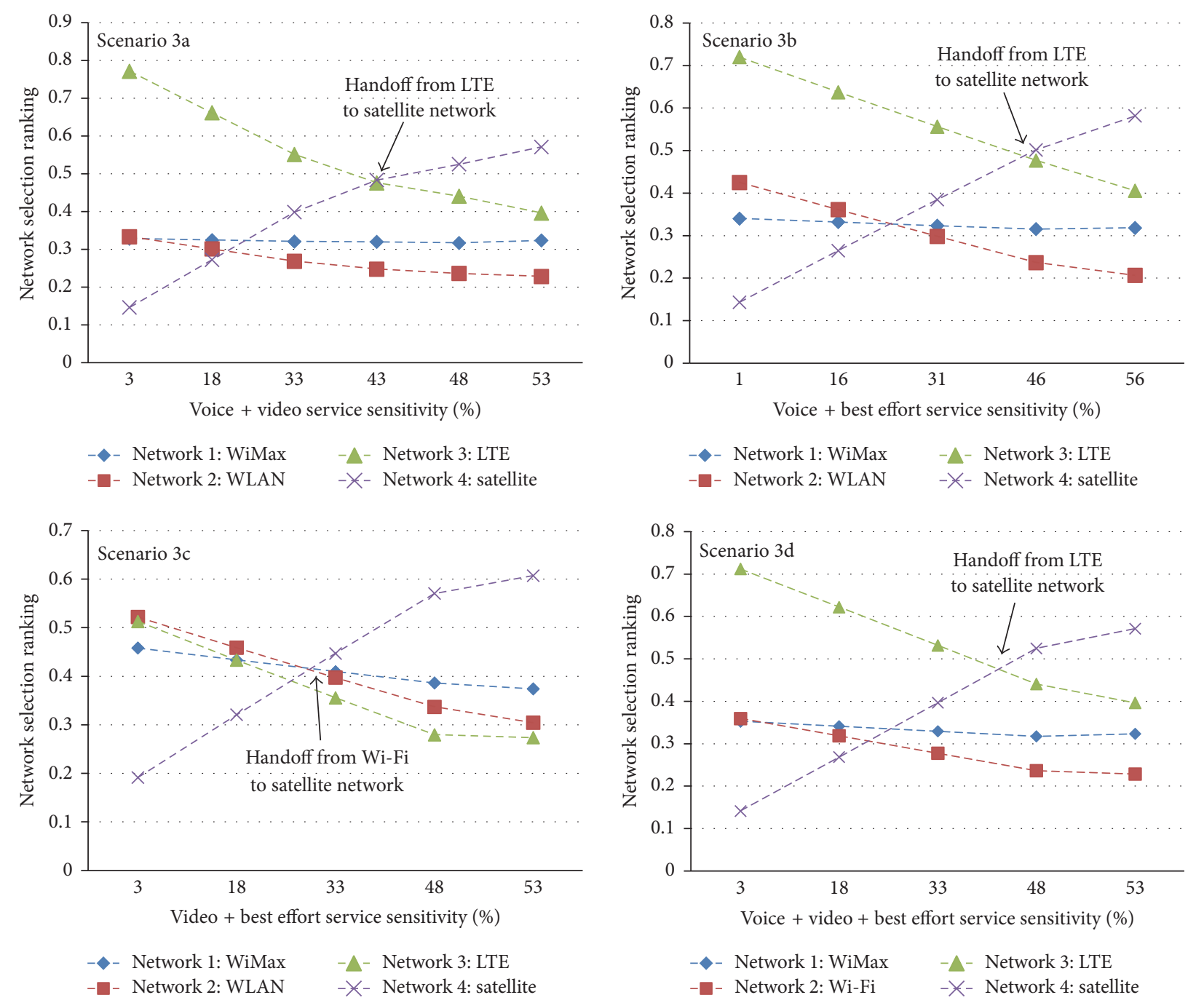

FIGURE 10: Impact of mobile network velocity on optimal network selection for spectrum handoff for internetwork spectrum handoff group of nonsafety services admitted by CR vehicular node. Scenario 3a: voice + video service; Scenario 3b: voice + best effort service; Scenario 3c: video + best effort service; Scenario 3d: voice + video + best effort service.

Case 2 (voice + best effort service). In Figure 9, Scenario $2 \mathrm{~b}$ and Table 6 show LTE is the optimal network for spectrum handoff for voice and best effort group of nonafety service. In Figure 10, Scenario $3 \mathrm{~b}$ shows that, with the increases in mobile network velocity, the network selection ranking decreases for LTE network and increases for satellite network.
Hence, there is spectrum handoff from LTE to satellite network with the increase in mobile network velocity.

Case 3 (video + best effort service). In Figure 9, Scenario $2 \mathrm{c}$ and Table 6 show WLAN is the optimal network for spectrum handoff for video and best effort group of nonsafety service. 


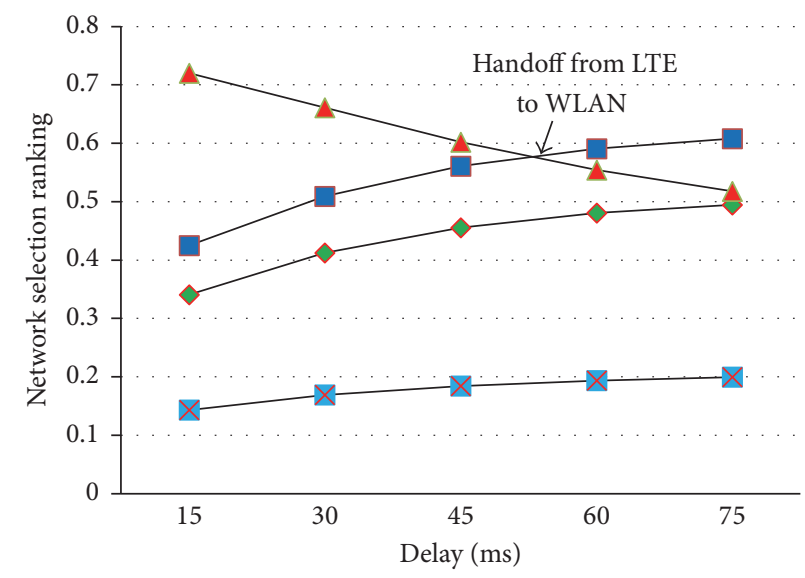

Scenario 4a: voice + best effort service

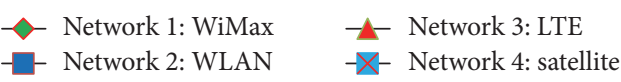

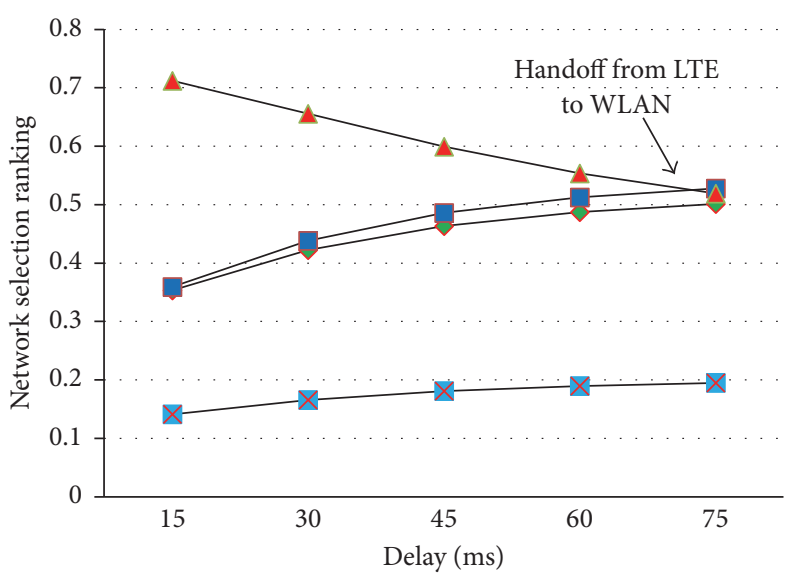

Scenario $4 \mathrm{~b}$ : voice + video + best effort service

Network 1: WiMax

Network 2: WLAN
-1- Network 3: LTE

$-\chi-$ Network 4: satellite

FIGURE 11: Effect of dynamic network environment, delay sensitive on optimal network selection for the internetwork spectrum handoff for group of nonsafety services; Scenario 4a: voice + best effort service; Scenario 3b: voice + video + best effort service.

In Figure 10, Scenario 3c shows that, with the increases in mobile network velocity, the network selection ranking decreases for WLAN and increases for satellite network. Hence, there is spectrum handoff from WLAN to satellite network with the increase in mobile network velocity.

Case 4 (voice + video + best effort service). In Figure 9, Scenario $2 \mathrm{~d}$ and Table 6 show LTE is the optimal network for spectrum handoff for voice, video, and best effort group of nonsafety service. In Figure 10, Scenario 3d shows that, with the increases in mobile network velocity, the network selection ranking decreases for LTE network and increases for satellite network. Hence, there is spectrum handoff from LTE to satellite network with the increase in mobile network velocity.

In brief, for all the group of nonsafety service, there is spectrum handoff from other networks to satellite network with the increase in mobile network velocity.

\subsection{Sensitivity to the Dynamic Network Environment on Opti- mal Network Selection for the Internetwork Spectrum Handoff for Group of Nonsafety Services}

Case 1 (delay sensitive). In Table 6, Scenario 2a, voice and best effort, and Scenario 2d, voice, video, and best effort group of nonsafety, show that LTE is optimal network for the spectrum handoff decision. If the delay attribute of LTE network increases due to the dynamic network environment, the optimal network for the spectrum handoff changes from LTE to WLAN as shown in Figure 11.

Case 2 (access price). In Table 6, Scenario 2c, video and best effort group of nonsafety, shows that WLAN is optimal network for the spectrum handoff decision. If the operator

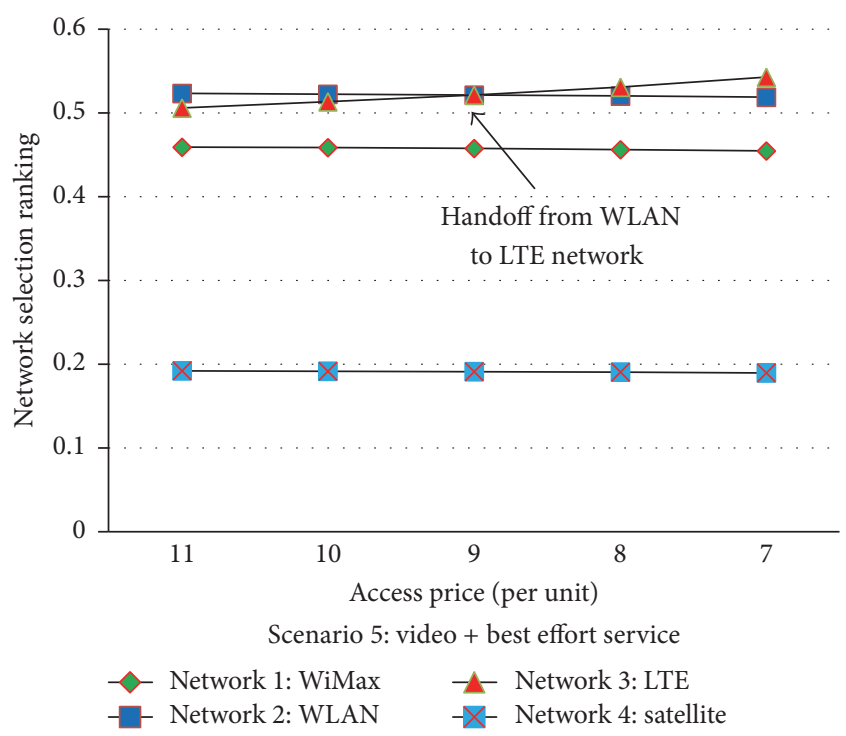

FIgURE 12: Access price sensitive dynamic network environment on optimal network selection for the internetwork spectrum handoff for group of nonsafety services; Scenario 5: video + best effort service.

of LTE network decreases its access price per unit to attract more number of users, the optimal network for the spectrum handoff changes from WLAN to LTE network as shown in Figure 12.

Case 3 (data rate). In Table 6, Scenario 2c, video and best effort group of nonsafety service, shows that WLAN is optimal network for the spectrum handoff decision. If data rate of WiMax network increases, then it startS to become attractive for video and best effort group of nonsafety service. 


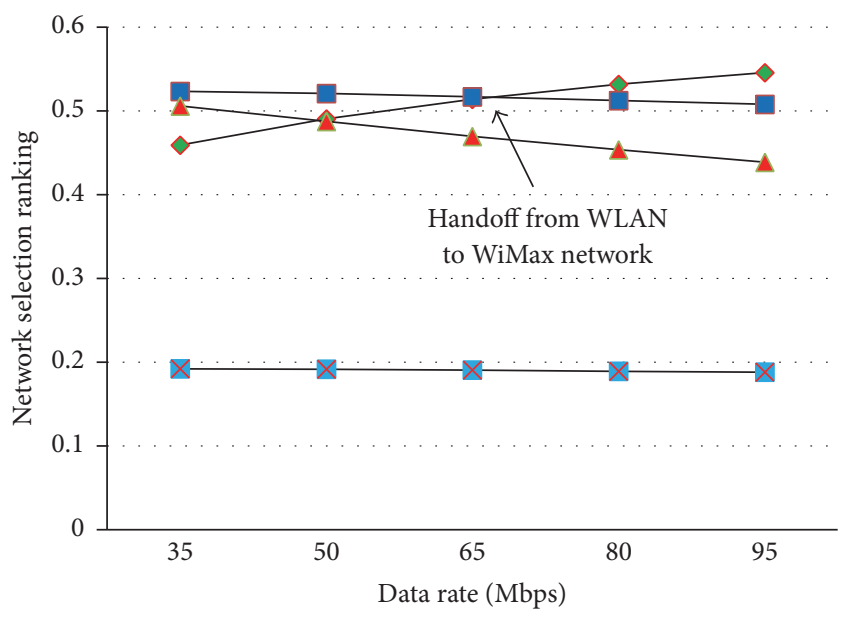

Scenario 6: video + best effort service

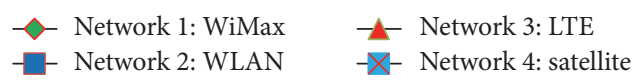

Figure 13: Data rate sensitive dynamic network environment on optimal network selection for the internetwork spectrum handoff for group of nonsafety services; Scenario 6: video + best effort service.

The optimal network for the spectrum handoff changes from WLAN to WiMax network as shown in Figure 13.

\section{Conclusion}

The multimode terminals have the capability to make group of call for two or more different classes of services simultaneously in next generation wireless network. This paper has proposed a spectrum handoff scheme for optimal network selection in NEMO based CR vehicular networks using multiple attributes decision making. The multimode CR vehicular nodes have the capability to make groups call for two or more types of nonsafety services simultaneously. The GRA and cost based MADM methods are used for optimal network selection for the spectrum handoff decision. The scheme is tested for its adaption to the various scenarios based on internetwork spectrum handoff. The obtained numerical results demonstrated that MADM methods are effective for the optimal network selection for spectrum handoff. The proposed scheme is also validated on mobile network velocity variation. It is found that when the mobile network velocity becomes high, change in the optimal network for spectrum handoff occurs significantly. Finally, the validation of the proposed scheme is also tested through the dynamic network environment such as delay sensitive, access price variation, and data rate variation. It is found that the optimal network for the spectrum handoff may change from one network to another, depending on the attribute variation in the dynamic network environment.

It is interesting to extend this spectrum handoff scheme for self-organizing NEMO based CR vehicular networks that learn about network conditions with knowledge and coordination. Furthermore, how to integrate this proposed scheme in vehicle assisted cross-layer handoff scheme is also an interesting issue.

\section{Competing Interests}

The authors declare that there is no conflict of interests regarding the publication of this paper.

\section{References}

[1] H. Bezabih, B. Ellingsaeter, J. Noll, and T. Maseng, "Digital broadcasting: increasing the available white space spectrum using TV receiver information," IEEE Vehicular Technology Magazine, vol. 7, no. 1, pp. 24-30, 2012.

[2] D. Das and S. Das, "A survey on spectrum occupancy measurement for cognitive radio," Wireless Personal Communications, vol. 85, no. 4, pp. 2581-2598, 2015.

[3] FCC, "Docket no. 03-322 Notice of proposed rule making and order," 2003, http://www.cs.ucdavis.edu/ liu/289I/Material/ FCC-03-322A1.pdf.

[4] B. Wang and K. J. R. Liu, "Advances in cognitive radio networks: a survey," IEEE Journal of Selected Topics in Signal Processing, vol. 5, no. 1, pp. 5-23, 2011.

[5] S. Haykin, "Cognitive radio: Brain-empowered wireless communications," IEEE Journal on Selected Areas in Communications, vol. 23, no. 2, pp. 201-220, 2005.

[6] K. Kumar, A. Prakash, and R. Tripathi, "Spectrum handoff in cognitive radio networks: a classification and comprehensive survey," Journal of Network and Computer Applications, vol. 61, pp. 161-188, 2016.

[7] J. Mitola III and G. Q. Maguire Jr., "Cognitive radio: making software radios more personal," IEEE Personal Communications, vol. 6, no. 4, pp. 13-18, 1999.

[8] J. Mitola III, "Cognitive radio architecture evolution," Proceedings of the IEEE, vol. 97, no. 4, pp. 626-641, 2009.

[9] J. Mitola III, "Cognitive radio for flexible mobile multimedia communications," Mobile Networks and Applications, vol. 6, no. 5, pp. 435-441, 2001.

[10] W. Jouini, C. Moy, and J. Palicot, "Decision making for cognitive radio equipment: analysis of the first 10 years of exploration," EURASIP Journal on Wireless Communications and Networking, vol. 2012, article 26, 2012.

[11] K. D. Singh, P. Rawat, and J.-M. Bonnin, "Cognitive radio for vehicular ad hoc networks (CR-VANETs): approaches and challenges," Eurasip Journal on Wireless Communications and Networking, vol. 2014, article 49, 2014.

[12] A. Prakash, S. Tripathi, R. Verma, N. Tyagi, R. Tripathi, and K. Naik, "Vehicle assisted cross-layer handover scheme in NEMObased VANETs (VANEMO)," International Journal of Internet Protocol Technology, vol. 6, no. 1-2, pp. 83-95, 2011.

[13] Q. Song and A. Jamalipour, "Network selection in an integrated wireless LAN and UMTS environment using mathematical modeling and computing techniques," IEEE Wireless Communications, vol. 12, no. 3, pp. 42-48, 2005.

[14] R. Verma and N. P. Singh, "GRA based network selection in heterogeneous wireless networks," Wireless Personal Communications, vol. 72, no. 2, pp. 1437-1452, 2013.

[15] G. Wei, "Grey relational analysis model for dynamic hybrid multiple attribute decision making," Knowledge-Based Systems, vol. 24, no. 5, pp. 672-679, 2011. 
[16] K. Kumar, A. Prakash, and R. Tripathi, "Context aware spectrum handoff scheme in cognitive radio vehicular networks," International Journal of Ad Hoc and Ubiquitous Computing, In press.

[17] V. Devarapalli, R. Wakikawa, A. Petrescu, and P. Thubert, "Network Mobility (NEMO) Basic Support Protocol," IETF RFC RFC3963 2005, https://www.ietf.org/rfc/rfc3963.txt.

[18] Z. Zhi-Jin, Z. Lu-Ping, and W. Hai-Quan, "Spectrum handoff based on adaptive weights adjustment," IET Communications, vol. 9, no. 5, pp. 674-680, 2015.

[19] K. L. Haldar, C. Ghosh, and D. P. Agrawal, "Dynamic spectrum access and network selection in heterogeneous cognitive wireless networks," Pervasive and Mobile Computing, vol. 9, no. 4, pp. 484-497, 2013.

[20] V. K. Tumuluru, P. Wang, D. Niyato, and W. Song, "Performance analysis of cognitive radio spectrum access with prioritized traffic," IEEE Transactions on Vehicular Technology, vol. 61, no. 4, pp. 1895-1906, 2012.

[21] Y. Xian, C. Xu, and H. Qian, "A grade-based spectrum handover mechanism in cognitive radio system," in Recent Advances in Computer Science and Information Engineering, Z. Qian, L. Cao, W. Su, T. Wang, and H. Yang, Eds., vol. 127, pp. 327-332, Springer, Berlin, Germany, 2009.

[22] S. Dhar, A. Ray, and R. Bera, "Cognitive vertical handover engine for vehicular communication," Peer-to-Peer Networking and Applications, vol. 6, no. 3, pp. 305-324, 2013.

[23] A. Prakash, R. Verma, R. Tripathi, and K. Naik, "A seamless handover scheme for vehicles across heterogeneous networks," International Journal of Communication Networks and Distributed Systems, vol. 8, no. 1-2, pp. 4-23, 2012.

[24] C. Shen, W. Du, R. Atkinson, and K. H. Kwong, "Policy based mobility \& flow management for IPv6 heterogeneous wireless networks," Wireless Personal Communications, vol. 62, no. 2, pp. 329-361, 2012.

[25] B. Bakmaz, Z. Bojkovic, and M. Bakmaz, "Network selection algorithm for heterogeneous wireless environment," in Proceedings of the 18th Annual IEEE International Symposium on Personal, Indoor and Mobile Radio Communications (PIMRC '07), pp. 1-4, Athens, Greece, September 2007.

[26] B. R. Chandavarkar and R. M. R. Guddeti, "Simplified and improved multiple attributes alternate ranking method for vertical handover decision in heterogeneous wireless networks," Computer Communications, vol. 83, pp. 81-97, 2016.

[27] M. Kassar, B. Kervella, and G. Pujolle, "An overview of vertical handover decision strategies in heterogeneous wireless networks," Computer Communications, vol. 31, no. 10, pp. 26072620, 2008.

[28] J. Márquez-Barja, C. T. Calafate, J.-C. Cano, and P. Manzoni, “An overview of vertical handover techniques: algorithms, protocols and tools," Computer Communications, vol. 34, no. 8, pp. 985997, 2011.

[29] M. Zekri, B. Jouaber, and D. Zeghlache, "A review on mobility management and vertical handover solutions over heterogeneous wireless networks," Computer Communications, vol. 35, no. 17, pp. 2055-2068, 2012.

[30] A. Afshari, M. Mojahed, and R. M. Yusuff, "Simple additive weighting approach to personnel selection problem," International Journal of Innovation and Technology Management, vol. 1, no. 5, pp. 511-515, 2010.
[31] W. Zhang, "Handover decision using fuzzy MADM in heterogeneous networks," in Proceedings of the IEEE Wireless Communications and Networking Conference (WCNC'04), vol. 2, pp. 653658, Atlanta, Ga, USA, March 2004.

[32] M. Behzadian, S. K. Otaghsara, M. Yazdani, and J. Ignatius, "A state-of the-art survey of TOPSIS applications," Expert Systems with Applications, vol. 39, no. 17, pp. 13051-13069, 2012.

[33] Y. S. Hussein, B. M. Ali, M. F. A. Rasid, A. Sali, and A. M. Mansoor, "A novel cell-selection optimization handover for longterm evolution (LTE) macrocellusing fuzzy TOPSIS," Computer Communications, vol. 73, pp. 22-33, 2016.

[34] S.-J. Yang and W.-C. Tseng, "Design novel weighted rating of multiple attributes scheme to enhance handoff efficiency in heterogeneous wireless networks," Computer Communications, vol. 36, no. 14, pp. 1498-1514, 2013. 


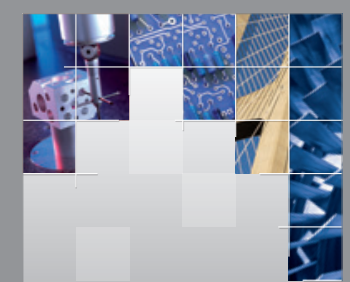

\section{Enfincering}
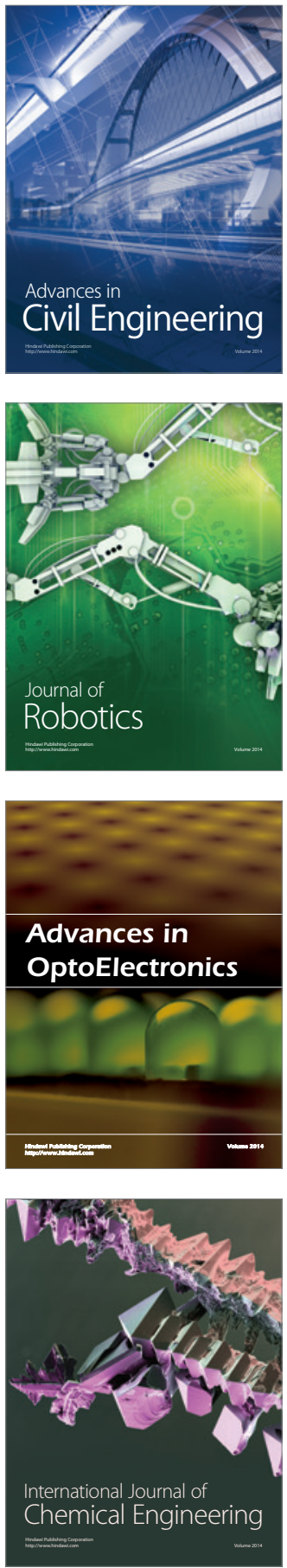

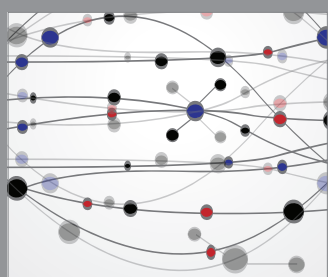

The Scientific World Journal

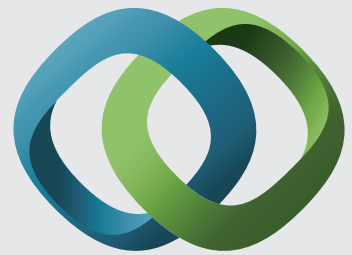

\section{Hindawi}

Submit your manuscripts at

https://www.hindawi.com
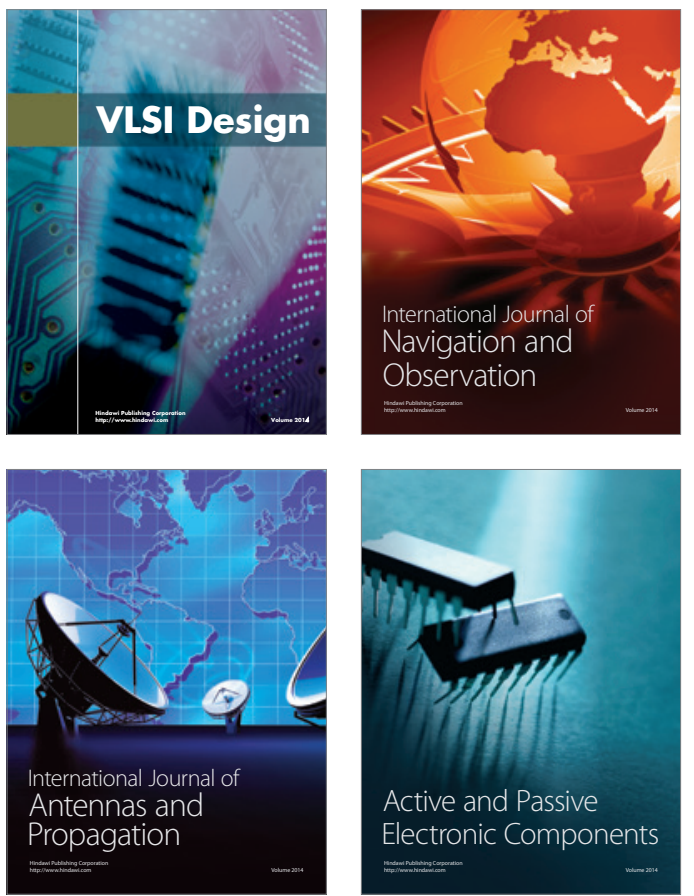
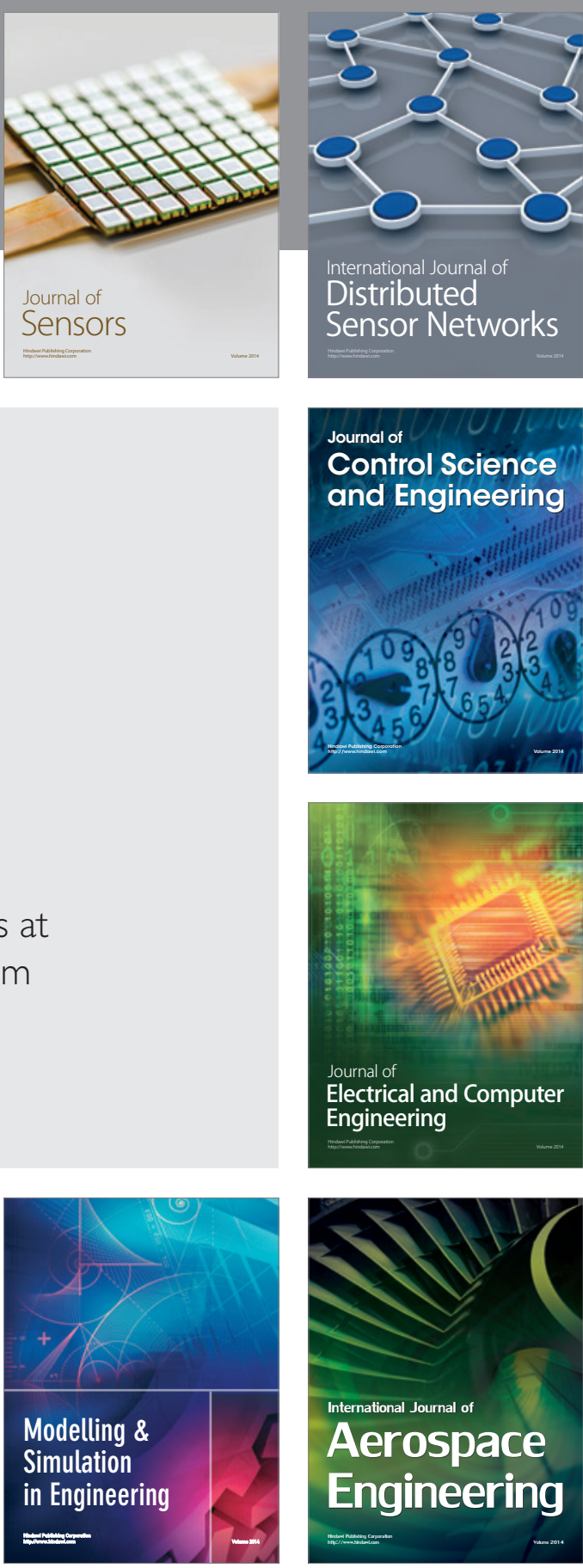

International Journal of

Distributed

Sensor Networks

$-$

Joumal of

Control Science

and Engineering
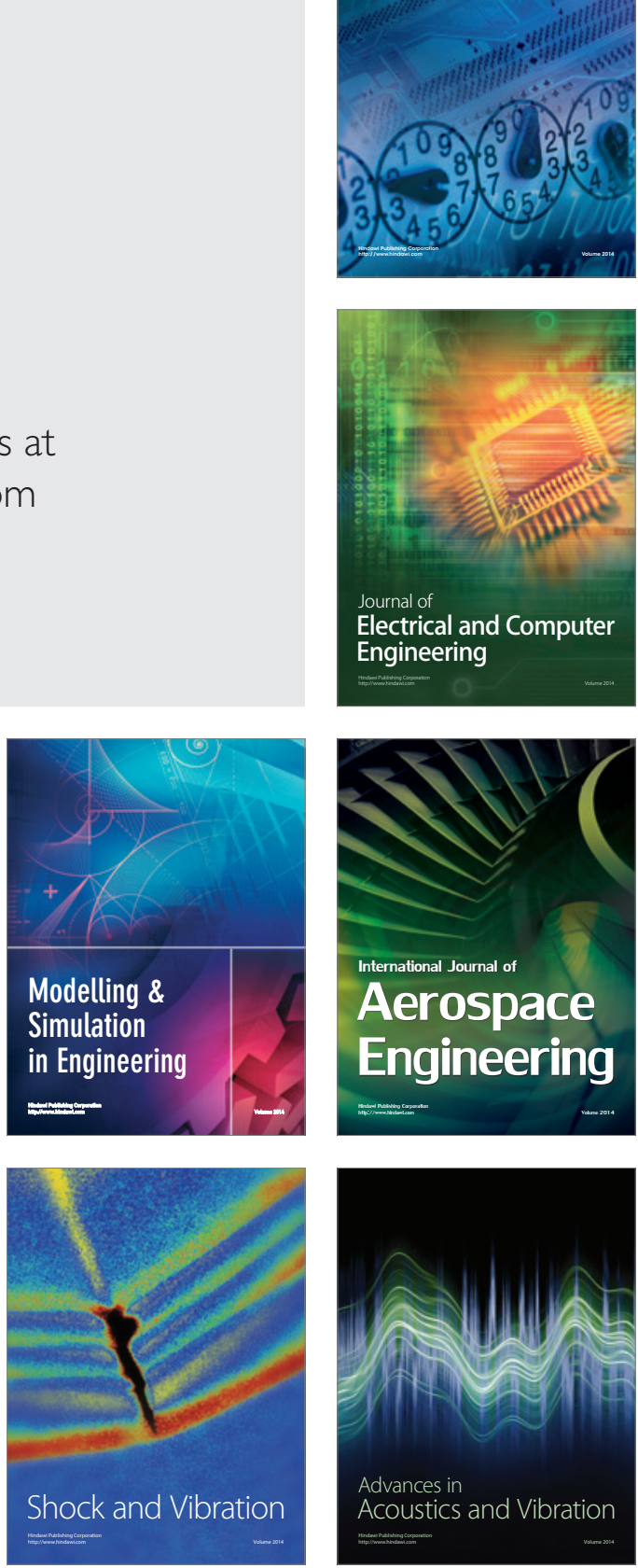